\title{
Clinical relevance of systematic phenotyping and exome sequencing in patients with short stature
}

\author{
Nadine N. Hauer, MSc ${ }^{1}$, Bernt Popp, MD ${ }^{1}$, Eva Schoeller, MSc ${ }^{1}$, Sarah Schuhmann, MD ${ }^{1}$, \\ Karen E. Heath, $\mathrm{PhD}^{2}$, Alfonso Hisado-Oliva, $\mathrm{BSc}^{2}$, Patricia Klinger, $\mathrm{PhD}^{3}$, Cornelia Kraus $\mathrm{PhD}^{1}$, \\ Udo Trautmann $\mathrm{PhD}^{1}$, Martin Zenker, $\mathrm{MD}^{4}$, Christiane Zweier, MD, PhD ${ }^{1}$, Antje Wiesener, $\mathrm{MD}^{1}$, \\ Rami Abou Jamra, MD ${ }^{5}$, Erdmute Kunstmann, MD ${ }^{6}$, Dagmar Wieczorek MD ${ }^{7,8}$, Steffen Uebe, PhD ${ }^{1}$, \\ Fulvia Ferrazzi, $\mathrm{PhD}^{1}$, Christian Büttner, $\mathrm{MSc}^{1}$, Arif B. Ekici, $\mathrm{PhD}^{1}$, Anita Rauch, $\mathrm{MD}^{9}$, \\ Heinrich Sticht, $\mathrm{PhD}^{10}$, Helmuth-Günther Dörr, MD ${ }^{11}$, André Reis, MD ${ }^{1}$ and Christian T. Thiel, MD ${ }^{1}$
}

\begin{abstract}
Purpose: Short stature is a common condition of great concern to patients and their families. Mostly genetic in origin, the underlying cause often remains elusive due to clinical and genetic heterogeneity.
\end{abstract}

Methods: We systematically phenotyped 565 patients where common nongenetic causes of short stature were excluded, selected 200 representative patients for whole-exome sequencing, and analyzed the identified variants for pathogenicity and the affected genes regarding their functional relevance for growth.

Results: By standard targeted diagnostic and phenotype assessment, we identified a known disease cause in only $13.6 \%$ of the 565 patients. Whole-exome sequencing in 200 patients identified additional mutations in known short-stature genes in $16.5 \%$ of these patients who manifested only part of the symptomatology. In
$15.5 \%$ of the 200 patients our findings were of significant clinical relevance. Heterozygous carriers of recessive skeletal dysplasia alleles represented $3.5 \%$ of the cases.

Conclusion: A combined approach of systematic phenotyping, targeted genetic testing, and whole-exome sequencing allows the identification of the underlying cause of short stature in at least $33 \%$ of cases, enabling physicians to improve diagnosis, treatment, and genetic counseling. Exome sequencing significantly increases the diagnostic yield and consequently care in patients with short stature.

Genet Med advance online publication 12 October 2017

Key Words: growth; phenotypic spectrum; short stature; skeletal dysplasia; whole-exome sequencing

\section{INTRODUCTION}

Growth retardation, a common condition leading to reduced height, is defined as the deviation of an individual's height of more than two SDs below the mean in the population or the estimated familial target height. ${ }^{1}$ The diagnosis is based on extent and type of growth retardation and other clinical signs and disorders. ${ }^{1,2}$ Conditions with growth retardation include inborn errors of development, which also pose a risk of various additional health issues like cancer, stroke, and cardiac defects. ${ }^{3-6}$ Along with these coexisting conditions, growth retardation might constitute a substantial emotional and clinical burden for affected individuals.

Short stature can be caused by nongenetic factors, such as nutrition, chronic systemic disorders, and emotional or psychosocial deprivation. ${ }^{7}$ Most forms of short stature, however, are based on genetic causes. Turner syndrome,
SHOX defects, mutations in genes affecting the growth hormone signaling pathway, or rare skeletal dysplasias are well-known causes. ${ }^{1,8-10}$ Nevertheless, the cause remains elusive in about $60-80 \%$ of patients, preventing early treatment of growth retardation and coexisting conditions as well as adequate genetic counseling. ${ }^{11,12}$

Human height is a polygenic trait with a heritability of about $80 \%{ }^{13}$ Several genome-wide association studies (GWAS) have identified some 700 common variants explaining $20 \%$ of height variation in the normal population and recently an additional $1.7 \%$ have been shown to be caused by rare and low-frequency coding variants. ${ }^{14,15}$ These studies probably explain some cases of short stature whereas recent studies suggest rare monogenic variants as the more common underlying cause. ${ }^{16-19}$ In absence of a specific clinical phenotype, unbiased genome-wide approaches are necessary

\footnotetext{
${ }^{1}$ Institute of Human Genetics, Friedrich-Alexander-Universität Erlangen-Nürnberg (FAU), Erlangen, Germany; ${ }^{2}$ Institute of Medical and Molecular Genetics (INGEMM) and Skeletal Dysplasia Multidisciplinary Unit (UMDE), Hospital Universitario La Paz, Universidad Autónoma de Madrid, IdiPAZ, and CIBERER, Madrid, Spain; ${ }^{3}$ Department of Orthopaedic Rheumatology, Friedrich-Alexander-Universität Erlangen-Nürnberg (FAU), Erlangen, Germany; ${ }^{4}$ Institute of Human Genetics, Otto-von-Guericke University Magdeburg, Magdeburg, Germany; ${ }^{5}$ Institute of Human Genetics, University of Leipzig, Leipzig, Germany; ${ }^{6}$ Institute of Human Genetics, University of Wuerzburg, Wuerzburg, Germany; ${ }^{7}$ Institute of Human Genetics, University of Essen, Essen, Germany; ${ }^{8}$ Institute of Human Genetics, University of Düsseldorf, Düsseldorf, Germany; ${ }^{9}$ Institute of Medical Genetics, University of Zurich, Zurich, Switzerland; ${ }^{10}$ Institute of Biochemistry, Friedrich-Alexander-Universität Erlangen-Nürnberg (FAU), Erlangen, Germany; ${ }^{11}$ Department of Pediatrics and Adolescent Medicine, Friedrich-Alexander-Universität Erlangen-Nürnberg (FAU), Erlangen, Germany. Correspondence: Christian T. Thiel (Christian.Thiel@uk-erlangen.de)
} 
to uncover such rare variants. We therefore investigated 565 patients with unexplained growth retardation by exhaustive clinical characterization followed by targeted genetic testing and exome sequencing in a representative subset of 200 patients.

\section{MATERIALS AND METHDOS}

\section{Patients}

We enrolled 565 consecutive patients and their families referred by local medical specialists for evaluation of growth retardation/short stature (Table 1, Supplementary Figure S1 online). Of these, 551 patients were of European, 13 of Asian, and 1 of Arab descent. At time of enrollment, 83\% of the patients were younger than 18 years. $81 \%$ presented with a height of 2 SDs below the age-related mean, whereas the remaining $19 \%$ were 2 SDs below the estimated target family height. Overall, $20 \%$ showed mild learning

Table 1 Clinical characteristics of included patients with idiopathic short stature

\begin{tabular}{|c|c|c|}
\hline Characteristic & $\begin{array}{c}\text { All } 565 \text { patients } \\
\text { No. (\%) }\end{array}$ & $\begin{array}{c}200 \text { Exome patients } \\
\text { No. }(\%)\end{array}$ \\
\hline \multicolumn{3}{|l|}{ Age group } \\
\hline$<4 y$ & $102(18)$ & $33(17)$ \\
\hline$>4 y$ & $463(82)$ & $167(83)$ \\
\hline Small for gestational age & $156(28)$ & $59(30)$ \\
\hline \multicolumn{3}{|l|}{ Short stature (SDs) } \\
\hline-2 to -3 & $270(48)$ & $99(50)$ \\
\hline-3 to -4 & $133(24)$ & $48(24)$ \\
\hline-4 to -5 & $34(6)$ & $17(8)$ \\
\hline$<-5$ & $19(3)$ & $9(4)$ \\
\hline Below est. height & $109(19)$ & $27(14)$ \\
\hline \multicolumn{3}{|l|}{ Short stature type } \\
\hline Isolated & $384(68)$ & $134(67)$ \\
\hline Syndromic & $181(32)$ & $66(33)$ \\
\hline \multicolumn{3}{|l|}{ Head circumference (SDs) } \\
\hline$>-2$ & $448(79)$ & $140(70)$ \\
\hline-2 to -3 & $60(11)$ & $26(13)$ \\
\hline-3 to -5 & $45(8)$ & $27(14)$ \\
\hline$<-5$ & $12(2)$ & $7(3)$ \\
\hline \multicolumn{3}{|l|}{ IQ } \\
\hline Normal & $450(80)$ & $160(80)$ \\
\hline $70-85$ & $115(20)$ & $40(20)$ \\
\hline \multicolumn{3}{|l|}{ Sex } \\
\hline Female & $349(62)$ & $122(61)$ \\
\hline Male & $216(38)$ & 78 (39) \\
\hline \multicolumn{3}{|l|}{ Bone age } \\
\hline Accelerated & $16(3)$ & $11(5)$ \\
\hline Normal & $27(5)$ & $19(10)$ \\
\hline Delayed & $125(22)$ & $68(34)$ \\
\hline Not available & $397(70)$ & $102(51)$ \\
\hline
\end{tabular}

a 200 representative patients from the 565 where the prior targeted clinical and diagnostic approach had not led to the identification of an underlying cause. These patients showed no statistical difference for each single or combined characteristic. disabilities and 21\% microcephaly. 30\% underwent bone age evaluation and of those $84 \%$ had either delayed or accelerated bone ages. All 565 patients underwent extensive prior endocrinological and diagnostic workup to exclude defects of the growth hormone pathway and organic causes of their growth deficit. All procedures were in accordance with the ethical standards of the FAU Erlangen-Nürnberg and the Helsinki Declaration. Detailed recruitment information is provided in the Methods section in the Supplementary Data.

\section{Systematic phenotyping and targeted testing}

On enrollment, all 565 patients received extensive genetic evaluation including syndromic and radiographic assessment by a clinical geneticist according to a standardized questionnaire. All information was included in a database based on known phenotype terms, followed by assessment of published information and discussion with a review board of experts in clinical genetics and dysmorphology (systematic phenotyping, Methods section in the Supplementary Data). ${ }^{20}$ Targeted genetic testing was applied based on known disease frequencies and phenotypic characteristics.

\section{Exome sequencing and variant assessment}

After exclusion of individuals from the group of 565 patients in whom our genetic targeted clinical and diagnostic approach had led to the identification of an underlying genetic cause of growth retardation, we selected a representative group of 200 patients (Figure 1, Table 1 and Supplementary Figure S1). These 200 patients showed no statistically significant difference from the remaining group of patients regarding age, height distribution, stature type, development, bone age, and sex (Table 1 and Supplementary Figure S1; selection and clinical description of the 200 individuals for exome analysis is provided in the Methods section in the Supplementary Data).

We performed whole-exome sequencing in this group of 200 patients -100 patients and both parents (trio analysis) and 100 patients (affected-only analysis) - after enrichment by SureSelect targeted capturing (Figure 1, Supplementary Figures S1-S2). Exomes were analyzed with our custom NGS Variant Analyzer tool, which involves the semiautomatic selection and data quality inspection of variants followed by the interpretation in relation to the reported phenotypic spectrum. The veracity and segregation in the families of selected variants were confirmed using Sanger sequencing.

All 200 patients were analyzed with respect to 1,000 known growth related genes derived from OMIM and MedGen databases (Supplementary Table S2). We considered causal all variants in known short stature-associated disease genes when the variant was predicted pathogenic or likely pathogenic referring to American College of Medical Genetics and Genomics (ACMG) criteria $^{21}$ and segregating with the phenotype in the family (Supplementary Data). All results were followed by a genotype-phenotype reevaluation. Detailed information about exome sequencing 


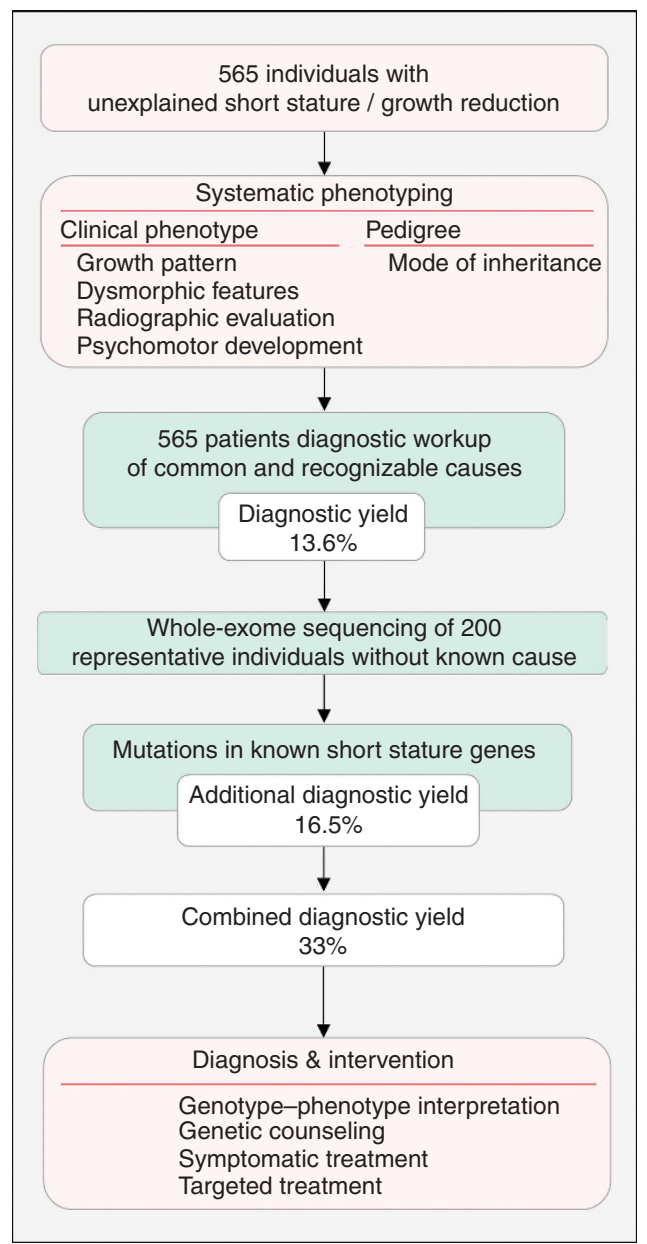

Figure 1 Flowchart gene discovery approach. We built a study group of 565 individuals with short stature or growth retardation (Table 1). Systematic phenotyping and targeted diagnostic testing of common and recognizable causes in all 565 patients led to a diagnostic yield of $13.6 \%$ (Supplementary Table 1). Detailed information about the systematic phenotyping is provided in the Methods section in the Supplementary Data. For 200 representative individuals where no underlying cause could be determined (Table 1), we performed whole-exome sequencing, which increased the diagnostic yield by $17 \%$ up to $33 \%$ by the identification of mutations in known short stature-associated genes (Table 2, Supplementary Tables 3-4). Genotype-phenotype re-evaluation confirmed that these patients present with only part of the characteristic symptomatology. The additional diagnostic yield already had direct effects in preventive measures, symptomatic and targeted treatment in $15.5 \%$ of the 200 exome-sequenced patients (Table 4 and Supplementary Table S6).

and interpretation of variants in known short-stature genes is provided in the Methods section in the Supplementary Data.

\section{Data availability}

Data for various analyses have been mentioned throughout the text and derived data supporting the findings of this study are available from the corresponding author upon request. Any other data associated with this study are available in the Supplementary Data. Additional methods are described in the Supplementary Data.

\section{RESULTS}

\section{Diagnostic yield of common and recognizable phenotypes in $\mathbf{5 6 5}$ individuals}

We could establish a diagnosis after systematic phenotyping in $13.6 \%$ of patients including disorders due to variants in 10 genes, imprinting defects, large chromosomal aberrations, and 24 distinct copy-number variants (Supplementary Table 1). Copy-number variants ranging in size from 0.1 to $14.2 \mathrm{Mb}$ were the most common cause of nonspecific syndromic short stature, identified in $6.9 \%$ of the patients. A syndromic form of short stature was suspected in 181 patients and confirmed in 26 of them $(4.6 \%$ of the 565 patients), mostly SHOX-related short stature and Silver-Russell syndrome. Turner syndrome or Turner syndrome variants were found in $1.6 \%$ of the patients and mutations in genes associated with skeletal dysplasia in $0.5 \%$. No diagnosis could be established in 491 (86.4\%) of the patients.

\section{Diagnostic yield of exome sequencing in $\mathbf{2 0 0}$ individuals}

To confirm the power of family-based exome sequencing in providing a diagnosis in individuals with growth retardation we selected a representative group of 200 families of the remaining patients with unexplained growth retardation (Figure 1, Table 1, and Supplementary Figure 1). We considered genes associated with growth phenotypes according to OMIM and MedGen databases and in these confirmed variants with regard to segregation and the specific patient's phenotype. Thereby, we identified 38 variants affecting 26 genes in 33 of 200 exome patients (16.5\% of the exome group; Table 2, Supplementary Figures S3-S27 and Supplementary Tables S2-S4). Of the 38 variants, $27(71 \%)$ were missense, 5 (13\%) frameshift, $4(11 \%)$ nonsense, and $2(5 \%)$ canonical splice-site variants. Twenty-nine of the 38 variants were not reported in the Exome Aggregation Consortium database, and nine were rare, with a maximum frequency of $2 \times 10^{-4}$. Following the ACMG guidelines, ${ }^{21} 16$ of the identified 38 variants (42\%) were classified as likely pathogenic and the remaining 22 (58\%) were defined as pathogenic variants (Materials and Methods section, Table 2, and Supplementary Table S4). The median height of these 33 patients was 2.7 SDs below the average height in the population, $21 \%$ showed microcephaly, and $41 \%$ were classified as syndromic (Supplementary Figure S1 and Supplementary Table S3). Modes of inheritance were autosomal dominant (65\%), autosomal recessive (19\%), and X-linked recessive (15\%) (Table 2, Supplementary Tables S3-S4). Pathway analyses of the affected proteins revealed that $58 \%$ of them are involved in the main functional categories of cartilage formation, chromatin modification, and Ras-MAPK signaling (Table 3).

We further wanted to know whether the selection of specific phenotypes or a combination of phenotypic features might reveal a higher diagnostic yield. A support vector machine approach resulted in $85.5 \%$ probability that it is not currently 

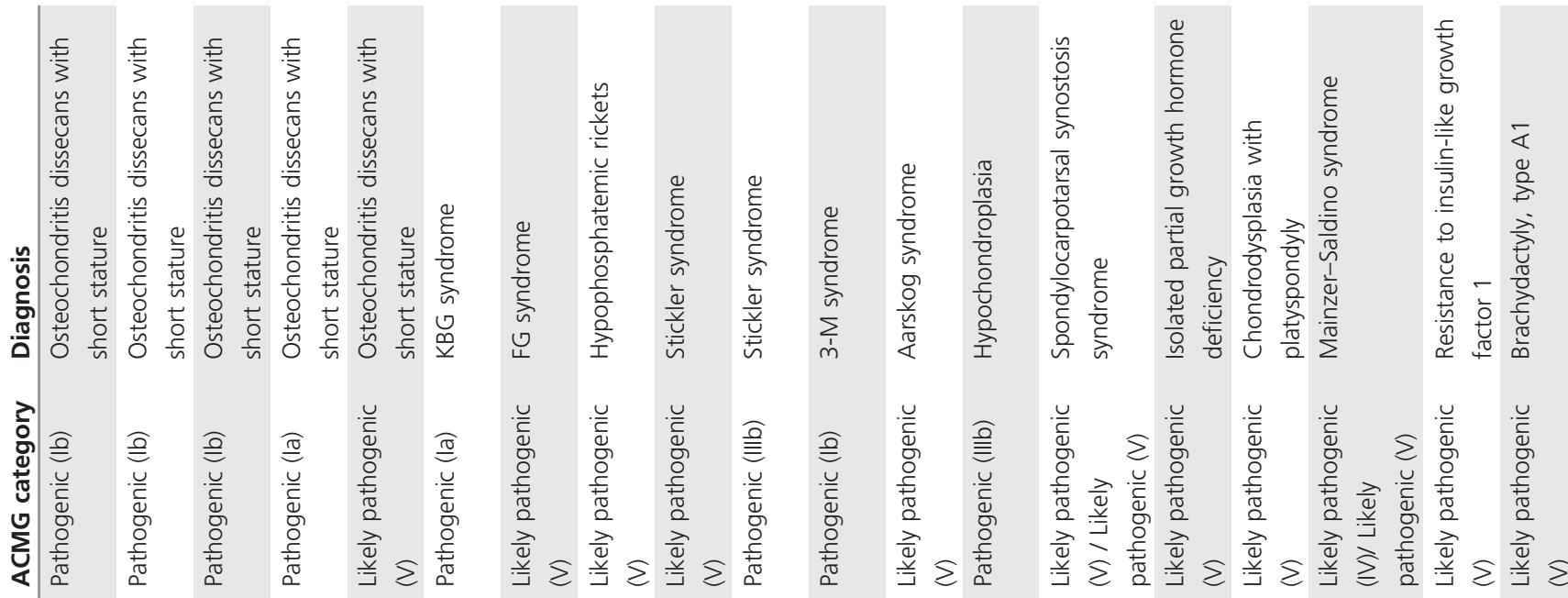

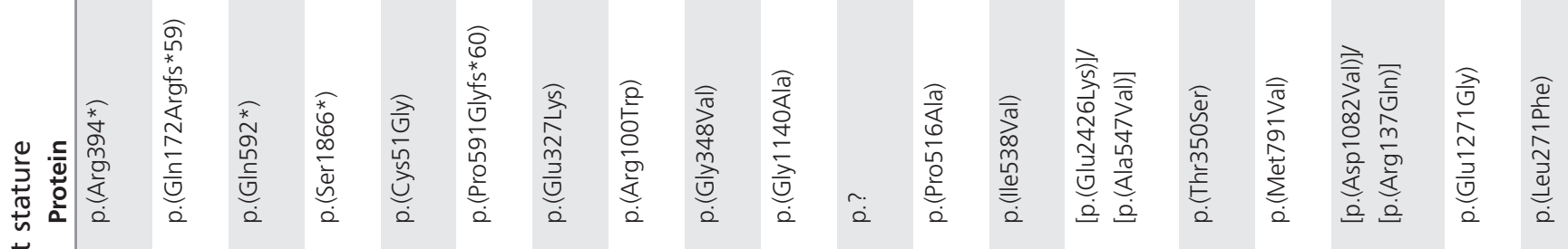

$\frac{t}{\frac{t}{n}}$

는

$\frac{0}{2}$

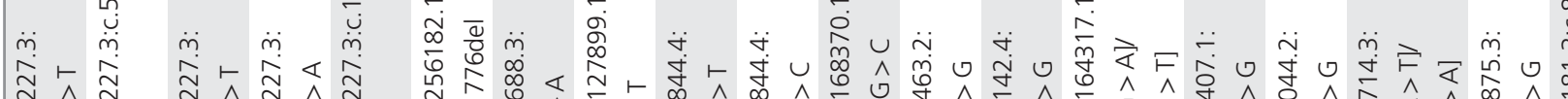

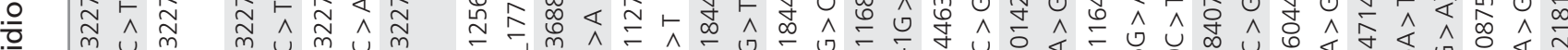

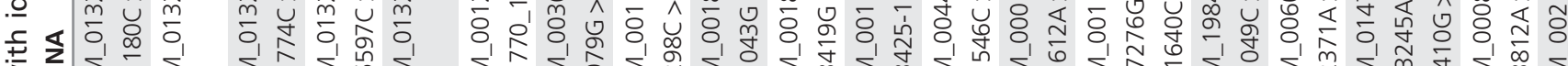

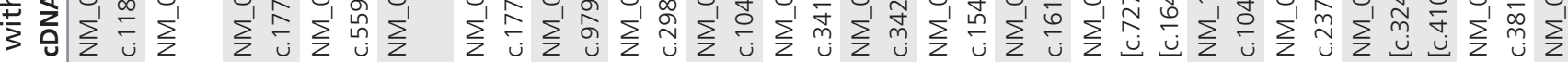

\section{$\frac{n}{\pi}$}

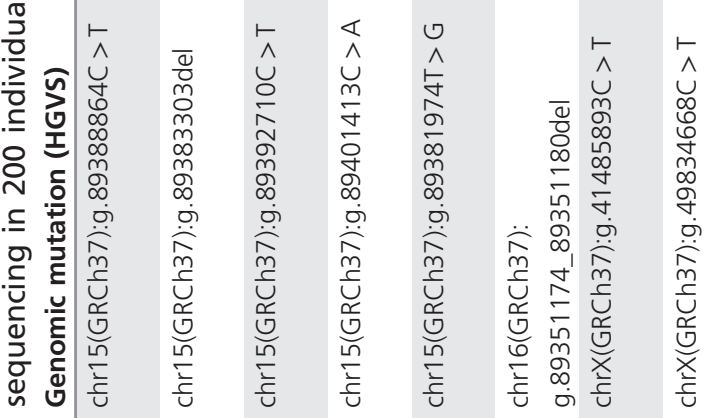
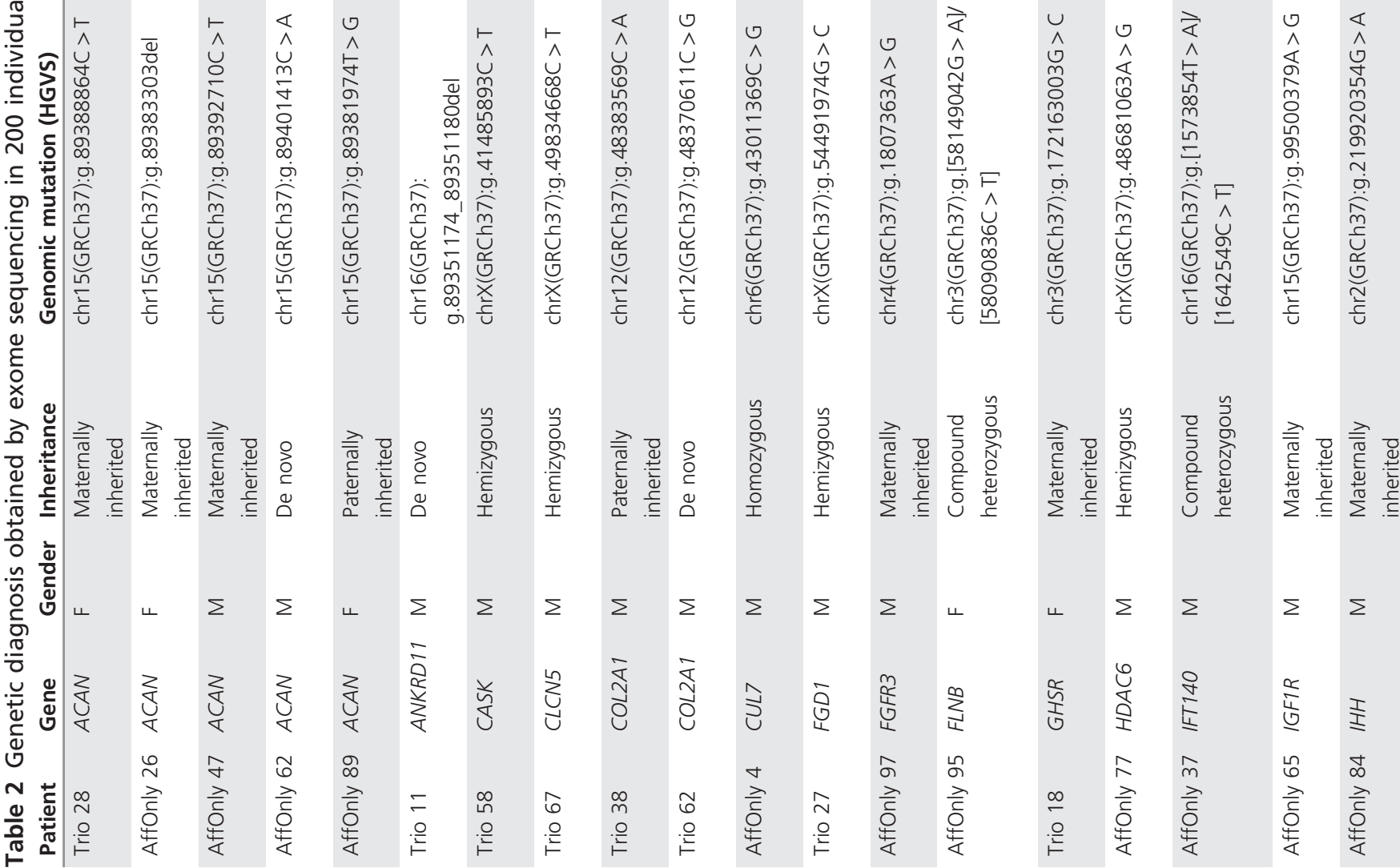

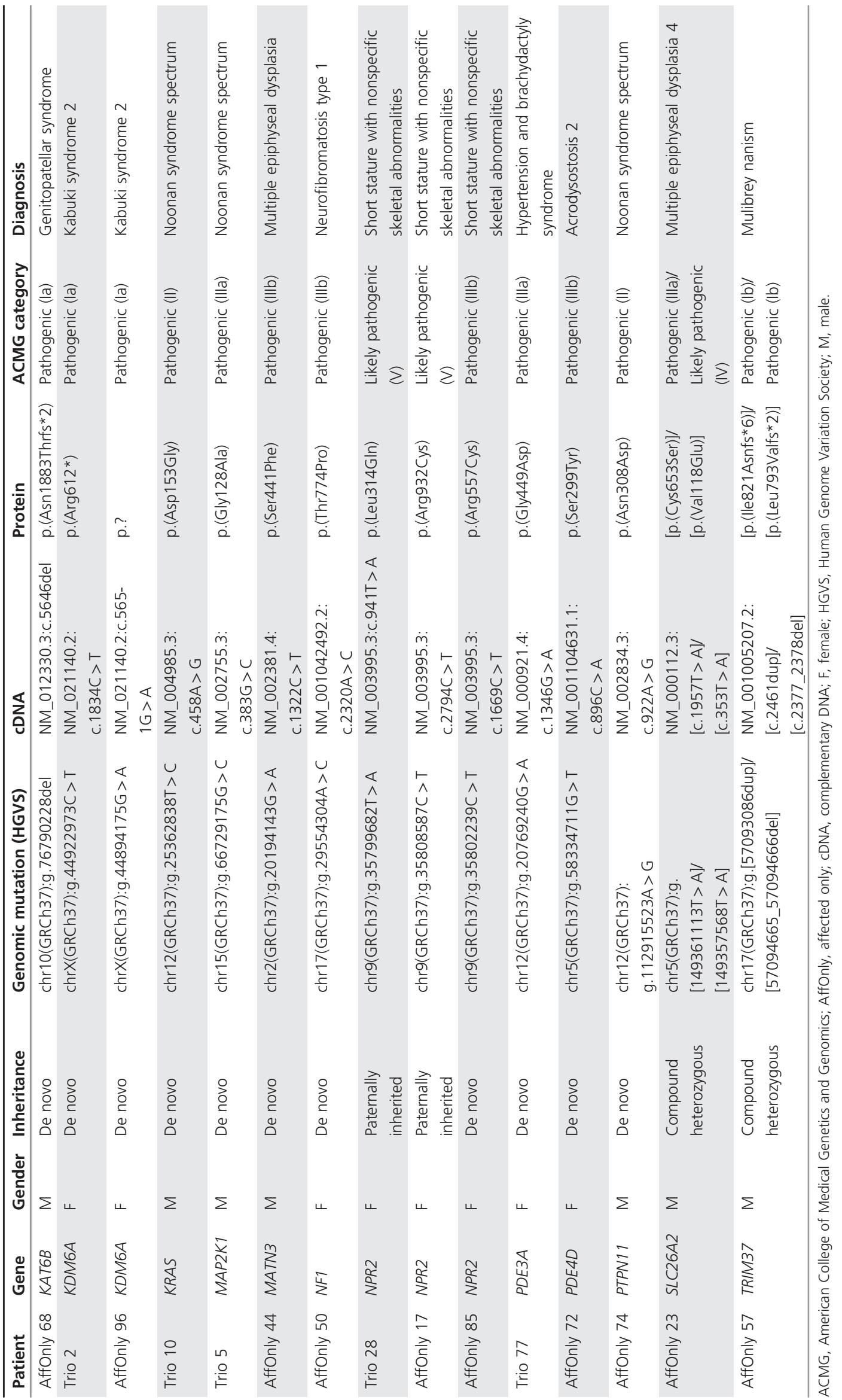
Table 3 Diagnostic yield of exome sequencing in 200 patients

\begin{tabular}{lcl} 
Main functional category $^{\text {a }}$ & No. of genes & Identified genes (no. of patients affected) \\
\hline Cartilage formation & 6 & ACAN (5), COL2A1 (2), FGFR3 (1), IHH (1), MATN3 (1), SLC26A2 (1) \\
\hline Chromatin modification & 5 & KDM6A (2), ANKRD11 (1), HDAC6 (1), KAT6B (1), TRIM37 (1) \\
Ras-MAPK pathway & 4 & KRAS (1), MAP2K1 (1), NF1 (1), PTPN11 (1) \\
Growth hormone-related pathway & 2 & GHSR (1), IGF1R (1) \\
Regulation of cytoskeleton & 2 & FGD1 (1), FLNB (1) \\
CAMP signaling pathway & 2 & PDE3A (1), PDE4D (1) \\
Centrosome/cilia formation & 2 & CUL7 (1), IFT14O (1) \\
mTOR signaling pathway & 1 & NPR2 (3) \\
Transcription regulation & 1 & $C A S K ~(1)$ \\
Renal regulation & 1 & $C L C N 5(1)$ \\
\hline
\end{tabular}

CAMP, cyclic adenosine monophosphate; MAPK, mitogen-activated protein kinase; mTOR, mammalian target of rapamycin.

aFrom DAVID functional annotation, OMIM, and KEGG.

possible to accurately predict the presence of a pathogenic mutation based on one or a combination of clinical subgroups (height, occipitofrontal circumference, intellectual disability, syndromic versus isolated phenotype, prenatal growth retardation, and accelerated/decelerated bone age) (Methods section in the Supplementary Data).

Overall, considering only genes previously associated with growth retardation, the diagnostic yield of $13.6 \%$ achieved with systematic phenotyping and targeted testing was raised to $33 \%$ by additional exome sequencing (Figure 1). The most commonly mutated known short stature-associated genes identified were ACAN (2.5\%), NPR2 (1.5\%), KDM6A (1\%), and COL2A1 (1\%) (Table 2 and Supplementary Tables S3-S4).

\section{Expansion of the phenotypic spectrum by exome sequencing}

Some of the mutations identified by exome sequencing are located in genes known to be associated with syndromic intellectual disability or skeletal dysplasia (Table 2 and Supplementary Tables S3-S5). One patient with a novel pathogenic KRAS missense mutation presented with proportionate short stature and learning disability illustrating the mild end of the Noonan syndrome spectrum disorders. ${ }^{22}$ Two patients with KDM6A mutations demonstrated the phenotypic variability of Kabuki syndrome. ${ }^{23,24}$ Neither showed any signs of developmental delay but they presented with part of the characteristic facial gestalt. Short stature is also an essential symptom of many skeletal dysplasias. ${ }^{9}$ We found mutations in FGFR3, COL2A1, and SLC26A2 in four patients presenting with no obvious specific skeletal involvement at initial clinical evaluation but consistent with the mild end of the spectrum of these entities upon reevaluation. Finally, we identified a previously unreported likely pathogenic hemizygous missense mutation in the calcium/calmodulin-dependent serine protein kinase gene $(C A S K)$ in a male patient with mild short stature and microcephaly but no intellectual disability. Loss-of-function CASK mutations underlie several forms of $\mathrm{X}$-linked intellectual disability syndromes and patients are reported to present with short stature. $^{25}$

\section{Carriers of recessive skeletal dysplasia mutations present with idiopathic short stature}

In $3.5 \%$ of the 200 exome patients we identified heterozygous mutations in two genes ( $A C A N, N P R 2)$ previously reported to cause the autosomal-recessive skeletal dysplasias spondyloepimetaphyseal dysplasia and acromesomelic dysplasia, respectively (Table 2, Supplementary Tables S3-S5). ${ }^{9,26}$ Heterozygous carriers consistently show idiopathic short stature without dysmorphic findings. ${ }^{26-30} \mathrm{We}$ found heterozygous mutations in ACAN in five and NPR2 in three patients who were not previously suspected to be carriers. The height of these patients ranged between -2.0 and -4.7 SDs (Supplementary Table S3). One patient carried both a variant in NPR2 and a variant in ACAN. This could indicate a blended phenotype as previously reported. ${ }^{31}$

\section{Additional relevance of exome sequencing results for clinical management}

Besides the increased diagnostic yield by exome sequencing in the 200 patients with idiopathic short stature, results with possible impact on treatment or additional preventive measurements occurred in 31 families (15.5\% of 200 exome individuals, Table 4 and Supplementary Table S6). This led to preventive measures for osteoarthritis (ACAN, CUL7, MATN3) and neoplasias (NF1, PTPN11, TRIM37), as well as orthopedic support and regular developmental evaluation in affected individuals from 23 families (11.5\%). Symptomatic treatment or screening for associated malformations (KAT6B, KRAS, MAP2K1, PTPN11), hearing loss (COL2A1, FLNB), or the risk for chronic kidney disease (IFT140, CLCN5) was recommended in nine families (4.5\%). A treatment with recombinant growth hormone or IGF1 has been shown to be beneficial especially in the individuals with defects of the growth hormone pathway. ${ }^{32-34}$ We identified mutations affecting genes of this pathway (GHSR, IGF1R) allowing specific treatment in three families (1.5\%). Interestingly, these have been missed by endocrine testing, but were confirmed by 


\begin{tabular}{|c|c|c|}
\hline Type & Symptom & Genes $^{a}$ \\
\hline \multirow[t]{6}{*}{ Preventive measures } & Osteoarthritis & ACAN, CUL7, MATN3 \\
\hline & Hearing loss & COL2A1 \\
\hline & Orthopedic symptoms & COL2A1, FGFR3, IHH, SLC26A2, HDAC6, FLNB \\
\hline & Developmental issues & KDM6A, ANKRD11, PDE4D, CASK, FGD1, PTPN11, NF1 \\
\hline & Bleeding diathesis & PTPN11 \\
\hline & Neoplasia & TRIM37, PTPN11, NF1 \\
\hline \multirow[t]{3}{*}{ Symptomatic treatment } & Hearing loss & COL2A1, FLNB \\
\hline & Multiple malformations & KAT6B, KRAS, MAP2K1, PTPN11 \\
\hline & Chronic kidney disease & IFT140, CLCN5 \\
\hline \multirow[t]{2}{*}{ Targeted treatment } & Growth hormone signaling pathway defects & GHSR, IGF1R, PTPN11 \\
\hline & Severe hypertension & PDE3A \\
\hline
\end{tabular}

anformation derived from GeneReviews and other publications (Supplementary Table S5).

growth hormone stimulation tests and therefore considered at least to contribute in part to the patient's growth phenotype. Also immediately relevant was the identification of a PDE3A mutation in one girl. Activating mutations in PDE3A led to hypertension and brachydactyly syndrome (MIM 123805) with the development of life-threatening hypertension later in life.$^{35} \mathrm{~A}$ targeted treatment with phosphodiesterase inhibitors is indicated due to its potentially significant impact on life expectancy. ${ }^{36}$ Finally, knowledge about the inheritance pattern will aid evaluation of the recurrence risk and improve genetic counseling of families.

\section{DISCUSSION}

Even though the heritability of short stature is considered high, the large number of genes implicated and the nonspecific clinical phenotype have led to poor yield of diagnostic genetic testing. . $^{3,16,18,19,37-39}$ To demonstrate the diagnostic outcome we enrolled 565 carefully characterized patients with short stature and their families and established an interdisciplinary systematic phenotyping with additional targeted gene testing (Figure 1). This led to a diagnostic yield of $13.6 \%$ including copy-number variants, chromosomal aberrations, and monogenic causes (Supplementary Table S1). We next performed unbiased exome sequencing in 200 representative patients with short stature of unknown origin (Table 1 and Supplementary Figure S1). The variant evaluation and classification in accordance with the ACMG guidlines ${ }^{21}$ led to the identification of mutations in known short stature-associated genes in 33 affected individuals (16.5\%) (Table 2, Supplementary Tables S3-S5). In a previous smaller study of 14 highly selected patients a diagnostic yield of $36 \%$ was reported, ${ }^{16}$ which might be explained by the study's strict inclusion criteria and smaller sample size. Genotype-phenotype re-evaluation confirmed that the individuals from our exome study were lacking most of the characteristic features of the entities, indicating that current descriptions are biased and that the phenotypic spectrum needs to be expanded. This is especially true for genes associated with syndromic forms of short stature and intellectual disability as well as skeletal dysplasias. There are parallels to the field of intellectual disability, also plagued by high genetic heterogeneity and unspecific clinical presentation, where both a similar mutation yield and inheritance modes were reported. ${ }^{40}$ However, in this study clinical subgrouping did not reveal any correlation between a specific phenotypic feature or combination of them and the probability to identify a mutation, thus supporting an unbiased approach such as exome analysis for all patients with idiopathic short stature.

One remarkable aspect was the detected frequency of heterozygous mutations in genes previously implicated in autosomal-recessive skeletal dysplasias (ACAN, NPR2), confirming a dosage effect of cartilage matrix proteins in growth development. ${ }^{26,27,29,30}$ In our exome study, ACAN was the most commonly mutated known short stature-associated gene with a frequency of $2.5 \%$ (Supplementary Table S2), whereas the previously reported most common single-gene defect affects SHOX with a frequency of $2.4 \%{ }^{8}$

An important aspect of the clinical application of wholeexome sequencing in short stature concerns prognosis, prevention, and treatment (Table 4 and Supplementary Table S6). In $11.5 \%$ of the families, the identification of the molecular cause of the disease by exome sequencing prompted further preventive action. The girl diagnosed with a mutation in PDE3A might benefit from current targeted treatment with phosphodiesterase inhibitors to reduce her high risk of life-threatening coronary artery disease and essential hypertension after puberty. ${ }^{35}$ Also $1.5 \%$ of the affected individuals became eligible for targeted treatment for growth retardation itself. Finally, 5\% of the patients could benefit from treatment or screening for associated malformations. None of these clinical applications were considered prior to genetic diagnosis by exome sequencing, but they have now been applied to the patients' care. 
In conclusion, we demonstrated that systematic phenotyping combined with targeted genetic testing and exome sequencing increases the diagnostic yield in short stature up to $33 \%$ with concomitant improvement in treatment and prevention. As height has a proposed heritability of about $80 \%$, and as the ongoing rate of discovery in other entities suggests, ${ }^{40}$ we expect that future identification of potential candidate genes, as well as their analysis in additional patients, will increase the diagnostic yield.

\section{SUPPLEMENTARY MATERIAL}

Supplementary material is linked to the online version of the paper at http://www.nature.com/gim

\section{ACKNOWLEDGMENTS}

This study was supported by DFG grants TH 896/3-3 and TH 896/3-4 and the Interdisciplinary Centre for Clinical Research of the Universität Erlangen-Nürnberg, (FAU) project F4. We thank all the patients and their families for participating in this project. We acknowledge the excellent technical support of Evelyn Galsterer and Farah Radwan. We also thank the Exome Aggregation Consortium and the groups that provided exome variant data for comparison. A full list of contributing groups can be found at http://exac.broadinstitute.org/about. This study makes use of data generated by the DECIPHER community. A full list of centers that contributed to the generation of the data is available from http://decipher.sanger.ac.uk and via e-mail from decipher@sanger.ac.uk.

\section{DISCLOSURE}

The authors declare no conflict of interest.

\section{REFERENCES}

1. Seaver $\mathrm{LH}$; on behalf of the American College of Medical Genetics (ACMG) Professional Practice and Guidelines Committee. ACMG practice guideline: genetic evaluation of short stature. Genet Med. 2009;11: 465-470.

2. Rauch A, Thiel $C T$, Schindler D, et al. Mutations in the pericentrin (PCNT) gene cause primordial dwarfism. Science. 2008;319:816-819.

3. Argente J. Challenges in the management of short stature. Horm Res Paediatr. 2016:85:2-10.

4. Mumtaz S, Yildiz E, Jabeen S, Khan A, Tolun A, Malik S. RBBP8 syndrome with microcephaly, intellectual disability, short stature and brachydactyly. Am J Med Genet A. 167A:3148-3152 2015.

5. Green J, Cairns BJ, Casabonne D, et al. Height and cancer incidence in the Million Women Study: prospective cohort, and meta-analysis of prospective studies of height and total cancer risk. Lancet Oncol. 2011;12:785-794.

6. Shimizu $Y$, Imano $H$, Ohira $T$, et al. Adult height and body mass index in relation to risk of total stroke and its subtypes: the circulatory risk in communities study. J Stroke Cerebrovasc Dis. 2014; 23:667-674

7. Amin N, Mushtaq T, Alvi S. Fifteen-minute consultation: the child with short stature. Arch Dis Child Educ Pract Ed. 2015;100: 180-184, 203.

8. Rappold GA, Fukami M, Niesler B, et al. Deletions of the homeobox gene SHOX (short stature homeobox) are an important cause of growth failure in children with short stature. J Clin Endocrinol Metab. 2002;87: 1402-1406.

9. Bonafe L, Cormier-Daire V, Hall C, et al. Nosology and classification of genetic skeletal disorders: 2015 revision. Am J Med Genet A. 2015;167A: 2869-2892.
10. Allen DB, Cuttler L. Clinical practice. Short stature in childhoodchallenges and choices. N Engl J Med. 2013;368:1220-1228.

11. Cohen $P$, Rogol $A D$, Deal $C L$, et al. Consensus statement on the diagnosis and treatment of children with idiopathic short stature: a summary of the Growth Hormone Research Society, the Lawson Wilkins Pediatric Endocrine Society, and the European Society for Paediatric Endocrinology Workshop. I Clin Endocrinol Metab. 2008;93: 4210-4217.

12. Waldman LA, Chia DJ. Towards identification of molecular mechanisms of short stature. Int J Pediatr Endocrinol. 2013;2013:19.

13. Visscher PM, Medland SE, Ferreira MA, et al. Assumption-free estimation of heritability from genome-wide identity-by-descent sharing between full siblings. PLoS Genet. 2006;2:e41.

14. Wood AR, Esko T, Yang J, et al. Defining the role of common variation in the genomic and biological architecture of adult human height. Nat Genet. 2014;46:1173-1186.

15. Marouli E, Graff M, Medina-Gomez C, et al. Rare and lowfrequency coding variants alter human adult height. Nature. 2017;542: 186-190.

16. Guo MH, Shen $Y$, Walvoord EC, et al. Whole exome sequencing to identify genetic causes of short stature. Horm Res Paediatr. 2014;82: 44-52.

17. Zahnleiter $D$, Uebe $S$, Ekici $A B$, et al. Rare copy number variants are a common cause of short stature. PLoS Genet. 2013;9:e1003365.

18. Zahnleiter D, Hauer NN, Kessler K, et al. MAP4-dependent regulation of microtubule formation affects centrosome, cilia, and Golgi architecture as a central mechanism in growth regulation. Hum Mutat. 2015;36:87-97.

19. Wit JM, Oostdijk W, Losekoot M, van Duyvenvoorde HA, Ruivenkamp CA, Kant SG. Mechanisms in endocrinology: novel genetic causes of short stature. Eur J Endocrinol. 2016;174:R145-R173.

20. Delude CM. Deep phenotyping: the details of disease. Nature. 2015;527: S14-S15.

21. Richards S, Aziz N, Bale S, et al. Standards and guidelines for the interpretation of sequence variants: a joint consensus recommendation of the American College of Medical Genetics and Genomics and the Association for Molecular Pathology. Genet Med. 2015; 17:405-424.

22. Schubbert S, Zenker M, Rowe SL, et al. Germline KRAS mutations cause Noonan syndrome. Nat Genet. 2006;38:331-336.

23. Lederer D, Grisart B, Digilio MC, et al. Deletion of KDM6A, a histone demethylase interacting with MLL2, in three patients with Kabuki syndrome. Am J Hum Genet. 2012;90:119-124.

24. Miyake N, Mizuno S, Okamoto $N$, et al. KDM6A point mutations cause Kabuki syndrome. Hum Mutat. 2013;34:108-110.

25. Moog U, Bierhals T, Brand K, et al. Phenotypic and molecular insights into CASK-related disorders in males. Orphanet J Rare Dis. 2015;10:44.

26. Tompson SW, Merriman B, Funari VA, et al. A recessive skeletal dysplasia, SEMD aggrecan type, results from a missense mutation affecting the C-type lectin domain of aggrecan. Am J Hum Genet. 2009;84:72-79.

27. Hisado-Oliva A, Garre-Vazquez Al, Santaolalla-Caballero F, et al. Heterozygous NPR2 mutations cause disproportionate short stature, similar to Leri-Weill dyschondrosteosis. J Clin Endocrinol Metab. 2015;100:E1133-E1142.

28. Vasques GA, Amano N, Docko AJ, et al. Heterozygous mutations in natriuretic peptide receptor-B (NPR2) gene as a cause of short stature in patients initially classified as idiopathic short stature. J Clin Endocrinol Metab. 2013;98:E1636-E1644.

29. Quintos JB, Guo MH, Dauber A. Idiopathic short stature due to novel heterozygous mutation of the aggrecan gene. I Pediatr Endocrinol Metab. 2015;28:927-932.

30. Bartels CF, Bukulmez $H$, Padayatti $P$, et al. Mutations in the transmembrane natriuretic peptide receptor NPR-B impair skeletal growth and cause acromesomelic dysplasia, type Maroteaux. Am J Hum Genet. 2004;75:27-34.

31. Posey JE, Harel T, Liu P, et al. Resolution of disease phenotypes resulting from multilocus genomic variation. N Engl J Med. 2017;376:21-31.

32. Richmond $E$, Rogol AD. Current indications for growth hormone therapy for children and adolescents. Endocr Dev. 2010;18:92-108.

33. Ali O, Wyatt D. Therapy of growth disorders. Curr Opin Endocrinol Diabetes Obes. 2011;18:3-8.

34. Franklin SL, Geffner ME. Growth hormone: the expansion of available products and indications. Pediatr Clin North Am. 2011;58: 1141-1165, $x$. 
35. Maass PG, Aydin A, Luft FC, et al. PDE3A mutations cause autosomal dominant hypertension with brachydactyly. Nat Genet. 2015;47: 647-653.

36. Movsesian M. Novel approaches to targeting PDE3 in cardiovascular disease. Pharmacol Ther. 2016;163:74-81.

37. Carmichael CM, McGue M. A cross-sectional examination of height, weight, and body mass index in adult twins. J Gerontol A Biol Sci Med Sci. 1995;50:B237-B244.

38. Silventoinen $\mathrm{K}$, Sammalisto $\mathrm{S}$, Perola $\mathrm{M}$, et al. Heritability of adult body height: a comparative study of twin cohorts in eight countries. Twin Res. 2003;6:399-408.

39. Silventoinen K. Determinants of variation in adult body height. J Biosoc Sci. 2003;35:263-285.

40. de Ligt J, Willemsen $\mathrm{MH}$, van Bon BW, et al. Diagnostic exome sequencing in persons with severe intellectual disability. $N$ Engl J Med. 2012:367:1921-1929
This work is licensed under a Creative Commons

Attribution-NonCommercial-NoDerivs

International License. The images or other third party material in this article are included in the article's Creative Commons license, unless indicated otherwise in the credit line; if the material is not included under the Creative Commons license, users will need to obtain permission from the license holder to reproduce the material. To view a copy of this license, visit http://creativecommons.org/licenses/ by-nc-nd/4.0/

(C) The Author(s) 2018 